\title{
Fast Approximation of Visibility Dominance Using Topographic Features as Targets and the Associated Uncertainty
}

\author{
Sanjay Rana
}

\begin{abstract}
An approach to reduce visibility index computation time and measure the associated uncertainty in terrain visibility analyses is presented. It is demonstrated that the visibility index computation time in mountainous terrain can be reduced substantially, without any significant information loss, if the line of sight from each observer on the terrain is drawn only to the fundamental topographic features, i.e., peaks, pits, passes, ridges, and channels. However, the selected sampling of targets results in an underestimation of the visibility index of each observer. Two simple methods based on iterative comparisons between the real visibility indices and the estimated visibility indices have been proposed for a preliminary assessment of this uncertainty. The method has been demonstrated for gridded digital elevation models.
\end{abstract}

\section{Introduction}

The visibility index, the amount of the terrain visible from a location, often computed in terms of the number of visible targets or related measures, is an important terrain parameter. Intrinsically, it is an indicator of the visual accessibility and visual dominance of the location, which are essential factors in determining the overall accessibility of the location. For this reason, visibility analysis of terrain is now a multidisciplinary and fertile topic for many practical applications. Applications of visibility analysis have varied from the planning of defense installations (e.g., watch towers, troop movements, flight paths, and air defense missile batteries-see Franklin et al. (1994)), communication/facilities allocation (e.g., TV/radio transmitters-see Lee (1991), De Floriani et al. (1994), and Kim et al. (2002)), landscape analysis (e.g., visibility graphssee O'Sullivan and Turner (2001)), and environmental modeling (e.g., terrain irradiation-see Wang et al. (2000a))

Two critical issues in visibility analyses are visibility index computation time and accuracy of the viewshed (area covered by the visible terrain). For simplicity, if we ignore the algorithmic and implementation-related (e.g., hardware) dependencies on the performance of a visibility analysis, then the computation time of visibility analyses is proportional to $O(o t)$, where $o$ is the number of observers (viewpoints) and $t$ is the number of targets on the terrain. Therefore, most optimized visibility index computation methods try to reduce the observer-target pair comparisons. It can be achieved by choosing a polyhedral terrain model (e.g., a triangulated irregular network or TIN—see De Floriani and Magillo (1994)) instead of a grid, and by using algorithmic heuristics (Franklin et al., 1994; Franklin, 2000; Wang et al., 2000b). It is unlike the exhaustive but time-consuming Golden Case, in which all the

Centre for Advanced Spatial Analysis, University College London, 1-19 Torrington Place, London WC1E 6BT, United Kingdom (s.rana@ucl.ac.uk). points, $n$, on the terrain are used as observers and targets. In other words, the visibility index computation time in a Golden Case is on the order of $O\left(n^{2}\right)$ because $o=t=n$. Accordingly, we regard all optimization approaches that reduce the Observers part of the computational load as the Reduced Observers Strategy. Similarly, the optimization approaches aiming to reduce the number of Targets (e.g., limiting the maximum visibility distance as in horizon culling) are regarded as the Reduced Targets Strategy. The visibility indices derived in a Golden Case could be referred to as the Absolute Visibility Indices (AVI) of the terrain points while the visibility indices based on any approximated and optimized visibility index computation are the Estimated Visibility Indices (EVI) of terrain points.

While the methods for modeling viewshed uncertainty are well known (e.g., see Fisher, 1991; Fisher, 1992; Fisher, 1993), the search for the optimization of visibility index computation time still goes on apace. In general, there is a compromise between performance and accuracy in any practical visibility index computation (Franklin et al., 1994). In this work, we propose an optimization of visibility index computation time by extending the observation of Lee (1992) that the fundamental topographic features, i.e., peaks, pits, passes, ridges, and channels, dominate the visibility of other ground locations and therefore could be good viewpoint sites. Based on this conclusion, we propose that, due to the exhaustive and optimal visual coverage provided by the fundamental topographic features, especially in mountainous uplands, they will also be the ideal set of targets to reduce the targets part of the visibility index computation load. The optimal nature of the topographic features is based on the consideration that they are generally fewer in number and have an objective geographic definition. In essence, we employ the Reduced Targets Strategy to reduce the visibility index computation time by drawing the line of sight (LOS) from observers to only fundamental topographic features. For brevity, we will use the term topographic features in place of fundamental topographic features. Interestingly, however, the reverse case is not necessarily true. In other words, the use of topographic features as the only observers, in order to estimate the overall visibility pattern, will not always guarantee a reliable visibility index pattern of the terrain. Another interesting argument is that whether a reduced number of random non-topographic feature targets could also provide reliable EVI (e.g., see Franklin et al., 1994). We will provide evidence which suggests that the reliability of the EVI in this case would depend upon the number of random

Photogrammetric Engineering \& Remote Sensing Vol. 69, No. 8, August 2003, pp. 881-888.

0099-1112/03/6908-881\$3.00/0 (C) 2003 American Society for Photogrammetry and Remote Sensing 
non-topographic feature targets and would vary with the nature of the terrain.

As explained above, our Reduced Targets Strategy reduces the visibility index of observers by an amount approximately equal to the non-topographic feature targets potentially visible to them. This kind of uncertainty, arising due to a sparse targets set, is closely similar to the uncertainties related to Object Generalization (Weibel and Dutton, 1999). To our knowledge, this kind of uncertainty has not been widely addressed in the visibility studies literature. In general, however, finding the location of visibly dominant observers has more practical use than their exact visibility indices (Franklin, 2000). Therefore, the aim is to evaluate whether the overall visibility index pattern is realistic, albeit abstracted. The following section proposes two simple methods based on an iterative comparison between the AVI and EVI of observers for assessing this uncertainty.

\section{Methodology}

In visibility analysis, a target is considered visible if an LOS from an observer can be drawn to it without its being obstructed by an intermediate point (an exception is provided by Wang (2000b), who used reference planes to establish the visible areas). The most common approach in previous Reduced Targets Strategy based optimization methods (e.g., see Franklin et al. (1994)) has been to draw the LOS from an observer to an arbitrary small number of randomly located targets on the terrain (Figure 1). In the current work, we propose that the visibility index of an observer be computed by drawing the LOS only to a topographic feature (Figure 1). Of course, the underlying assumption of this proposal is that the terrain is not devoid of topographic features. This is true for mountainous terrain except in upland plateaus, although in which case the visibility indices will be mostly similar. Our methodology for the computation of visibility indices using topographic feature targets consists of three steps: (1) extract the topographic features, (2) compute the visibility index of each point using the topographic features as targets, and (3) assess the uncertainty in the visibility index.

\section{An Experiment}

Step 1-Extraction of Topographic Features

Many approaches have been proposed for the automated extraction of topographic features from DEMs and TINs (Greysukh, 1967; Peucker and Douglas, 1975; Evans, 1979;

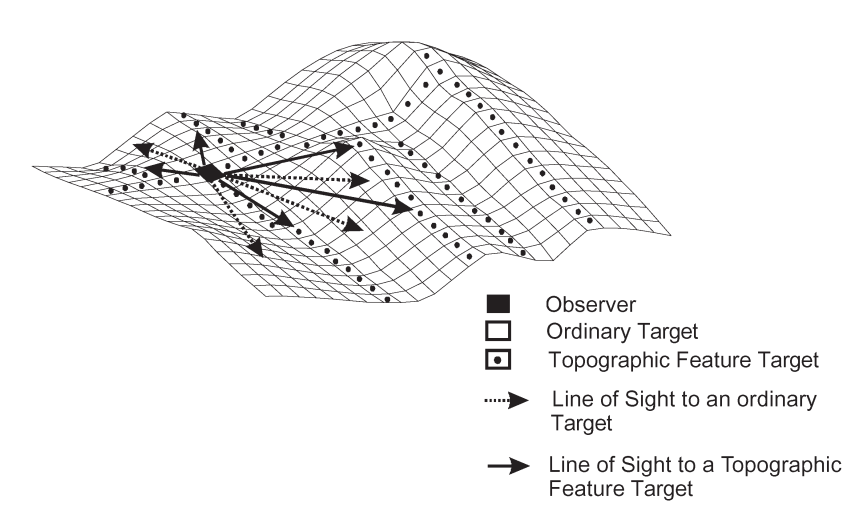

Figure 1. Two types of observer-target relationships in the Reduced Targets Strategy, i.e., line of sights drawn to random non-topographic feature targets and line of sights drawn to topographic features, i.e., peaks, passes, pits, ridges, and channels.
Takahashi et al., 1995; Wood, 1998). A detailed treatment of this topic is beyond the scope of this work. We decided to use the extraction method of Wood (1998), based on the advantages he outlined against the other methods and partly due to its easy availability in the user-friendly freeware software LandSerf.

It is clear that the success of our Reduced Targets Strategy depends upon the accuracy of the non-trivial topographic feature classification. It is well known that most automated topographic feature extraction methods are vulnerable to the noise in the DEM (Jenson and Domingue, 1988) and, most importantly, have scale dependency limitations (Wood, 1999). While smoothing the DEM before extracting the features can eliminate the first limitation, the latter seems to remain a difficult conceptual problem yet to be completely solved. Due to scale dependency, the automated feature extraction identifies features only at a certain scale (e.g., features of a fixed geographic extent), while features at other scales remain undetected. Therefore, the assessment of an appropriate scale for the particular DEM requires iterating through a number of feature extraction scales (e.g., in LandSerf, one could achieve this by iterating with a different window or kernel sizes for the feature extraction and visual verification).

Finally, although the fundamental topographic features are a significantly reduced number of targets, there may still be too many for certain terrains, e.g., large desiccated DEMs, and thus lead to a long visibility index computation time. Two simple ways of reducing the number of topographic features are to (1) resample the topographic features set by a certain skip interval and (2) limit the topographic features to certain scales. A detailed treatment of the sampling methods is beyond the scope of this paper but we will demonstrate the use of the first method later.

\section{Step 2-Visibility Analysis}

The study areas for the current work are the 100-m resolution DEMs of the Cairngorms (5548 points) in Scotland (Figure 2a) and the central part of the Isle of Man (16335 points) (Figure 3a). Note that this methodology could also be easily applied to an irregular terrain model such as a TIN. The visibility analysis was carried out in ArcView GIS developed by ESRI, and all the parameters were the defaults of the Visibility Request in ArcView. In the experiment, the observer eye level is at $1 \mathrm{~m}$ above the local ground level and the targets are at local ground level. The observer is capable of seeing from ground zero to infinity (i.e., no horizon culling), across the full range of azimuths, and from the zenith to nadir. The experiments were done on a 1-GHz Intel-Pentium processor-based personal computer, with $256 \mathrm{MB}$ RAM. We recorded the CPU time taken by ArcView for each visibility computation.
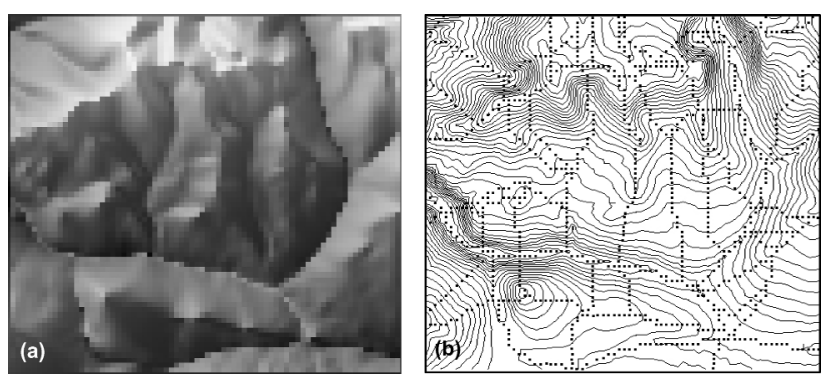

Figure 2. (a) Hill-shaded DEM of S.E. Cairngorm Mountains, Scotland. The minimum elevation is $395 \mathrm{~m}$ and the maximum elevation is $1054 \mathrm{~m}$. (b) 910 topographic feature targets. 

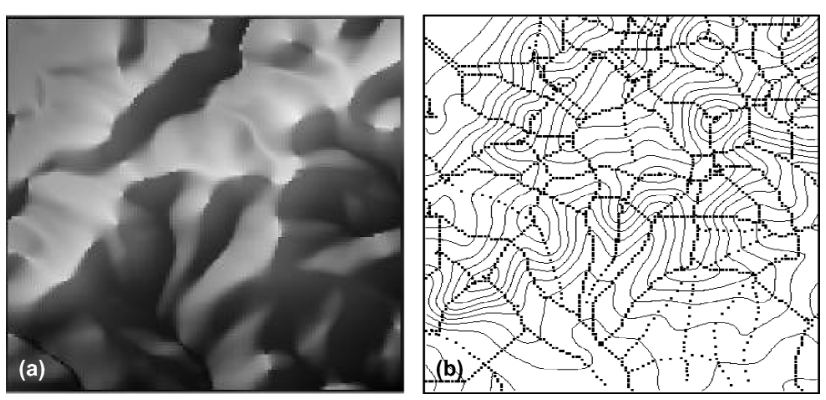

Figure 3. (a) Hill-shaded DEM of Central Isle of Man. The minimum elevation is $0 \mathrm{~m}$ and the maximum elevation is $553 \mathrm{~m}$. (b) 2007 topographic feature targets.

\section{Step 3-Uncertainty Assessment}

The only previous example known to us, which dealt with the estimation of uncertainty in a similar Reduced Targets Strategy, is that of Franklin et al. (1994). They compared the visibility indices of an arbitrary number of spatially distributed points in the terrain, computed from their exhaustive R2-visibility algorithm (similar to our Golden Case), with their optimized methods. Though the results are encouraging, their sampling methods (i.e., the selection of the test points) could not be regarded as formal and objective for two reasons. First, because there is no prior knowledge about the statistical distribution of the visibility pattern, it is not possible to estimate the number of random points required to fully capture the sensitivity of the visibility index distribution of a terrain. However, the choice of the number of random points is critical, because it will dictate our computation time. Second, because visibility is a directional property, then the spatial location of random points could bias the uncertainty estimation. Later we provide examples that suggest that the visibility pattern is highly dependent upon the spatial distribution and number of the random points.

We propose the following two methods for the uncertainty assessment based on a slight modification of the Franklin et al. (1994) methods:

Method 1: Spatial Correlation between AVI and EVI In this method, an assessment of the overall visibility pattern indicated by the EVI of the terrain points is done in the following two ways:

\section{Type 1: AVI vs. EVI of the Topographic Feature Observers-}

- Compute the AVI of the topographic features by drawing the LOS from each topographic feature to all the terrain points. Normalize the AVI and EVI of the topographic features, by scaling them between their respective minimum and maximum indices, in order to suppress the effect of target sample size on the indices. The normalization also reveals the visibility dominance of the observers.

- Calculate the correlation coefficient between the AVI and EVI of the topographic features. The correlation coefficient should suggest the similarity between the two visibility patterns. This method is similar to Franklin et al. (1994) except that the definition of our test points is objective and more natural. However, statistically it remains only an approximate test, especially when using exceptional terrains, where the topographic features are not distributed uniformly across the terrain.

\section{Type 2: AVI vs. EVI of Random Observers-}

Unlike the Type 1 method, this method is relatively more exhaustive but also more time-consuming. It is an abridged form of the Monte-Carlo method of uncertainty modeling and involves an iterative comparison between the AVI and EVI of a set of random observers but with the important exception that no subsequent model parameter estimation is done in this method. The steps are as follows:

(1) Estimate the number of observers to be distributed randomly on the terrain: As mentioned before, because there is no prior knowledge about the AVI distribution, it is non-trivial to determine the optimal number of random observers sufficient to capture the visibility pattern. We propose, without formal proof, that randomly placed observers, equal in number to the number of unique EVI, would be sufficient if we assume that

- No part of the study area is hidden from the topographic features. Thus, a histogram of the EVI (computed using topographic features) represents unique viewsheds, and

- Random observers do not form clusters.

In other words, with these assumptions, we suppose that each viewshed will be assigned at least one test-observer site.

(2) Distribute a number of random observers equal to the number of unique EVI, found in step (1), spatially across the terrain. We used the Random Point Generator ArcView Extension developed by Jennes (2001).

(3) Compute the AVI of the random observers by drawing the LOS to all the points on the terrain. Normalize the AVI and EVI of the random observers as previously done.

(4) Calculate the correlation coefficient between the AVI and EVI of the random observers.

(5) Repeat steps 2 through 4 a number of times. Again, due to the lack of any prior information about the statistical distribution of the AVI, statistically it is difficult to decide upon a specific number of iterations. In a practical exercise, it would ultimately depend upon the amount of time available to the researcher for the experiment.

(6) Choose the lowest and highest correlation coefficient as indicators for the worst- and the best-case approximation of AVI.

\section{Method 2: Error in the Estimated Visibility Indices}

In the previous methods, the AVI to EVI correlation coefficients give an indication of the reliability of EVI in representing the spatial pattern of visibility dominance. However, these do not reveal the amount of approximation in the EVI. A simple method for measuring the uncertainty in the EVI is an average ratio of EVI over AVI, as follows:

$$
\text { Average Error }(\%)= \pm \frac{\sum_{i=1}^{n} \frac{\left|x_{i}^{\prime}-x_{i}\right|}{x_{i}}}{n} \times 100 \ldots
$$

where $x_{i}^{\prime}$ is the normalized EVI of an observer $i, x_{i}$ is the normalized AVI, and $n$ is the number of observers. Note that normalized AVI and EVI are used to ensure that the approximation in visibility dominance is revealed.

\section{Significance of the Topographic Features}

As mentioned in the introduction, a Reduced Targets Strategy based on a small number of random non-topographic feature targets could also reduce the visibility index computation

time. Therefore, in order to validate the uniqueness and benefit of our choice of targets, we wanted to ensure that they would be better than the same number of random targets spatially distributed across the terrain. One of the ways of verifying the significance of the topographic features as targets is to compare the quality of the visibility pattern produced by an equal and decreasing number of topographic feature targets and the random targets. In this work, we used the skip interval method of generalizing our topographic feature set and gradually kept increasing the skip interval. Some other suitable guides for the minimum number of test points could be the number of point topographic features (peaks, pits, and passes), a satisfactory level of accuracy, and the maximum permissible computation time.

For each set of topographic feature targets, we generated four sets of equal numbers of random targets. The quality of 

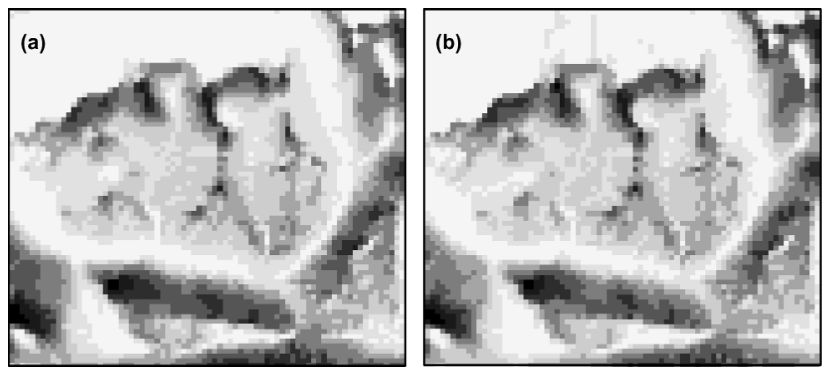

Figure 4. Comparison between the (a) Golden case based visibility dominance and (b) topographic features based estimated visibility dominance in the Cairngorm study area. Darker colored areas have more visual dominance than lighter colored areas.

the visibility dominance pattern produced from the topographic feature targets was then compared with the one produced from the random targets. The comparison was based on the two types of uncertainty estimation methods described in the previous section.

\section{Results}

\section{Automated Extraction of the Topographic Features}

After iterating with various window (kernel) sizes followed by visual inspection, we found that the 5 by $5(500 \mathrm{~m}$ by $500 \mathrm{~m}$ ) window and 3 by $3(300 \mathrm{~m}$ by $300 \mathrm{~m})$ window are suitable for extracting most linear (ridge, channel) and point (peak, pit, pass) topographic features, present in the Cairngorm (Figure 2b) and Isle of Man (Figure 3b) DEM, respectively, where 910 and 2007 topographic features have been extracted as the optimal targets. However, as mentioned previously, the number of topographic features extracted from the DEM depends upon the size of the filter window. Therefore, different window sizes will produce different numbers of topographic features. In future work, it would be interesting to investigate the change in the EVI pattern and its correlation with the AVI with varying extraction scales.

\section{Visibility Analysis and Uncertainty Assessment}

Because our study areas are small, we have been able to obtain the Golden Case visibility patterns of our study areas (Figures 4a and 5a). These visibility index patterns are now the ideal standards, i.e., the AVI. The visibility indices have been normalized (as previously) to assess the relative visibil-
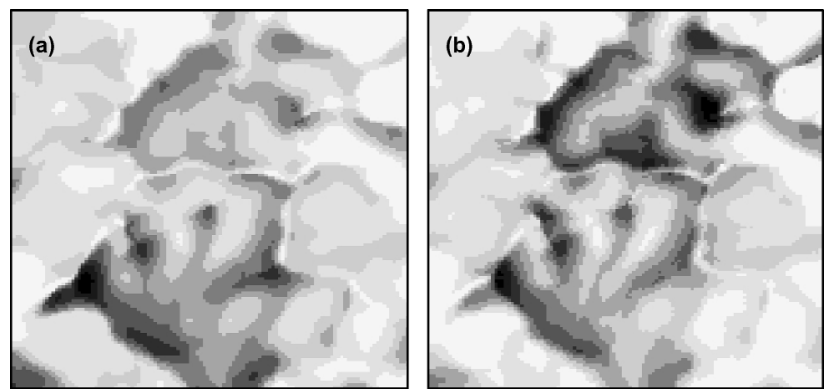

Figure 5. Comparison between the (a) Golden case based visibility dominance and (b) topographic features based estimated visibility dominance in the Isle of Man study area. Darker colored areas have more visual dominance than lighter colored areas. ity dominance of the points in the visibility pattern. Figures $4 \mathrm{~b}$ and $5 \mathrm{~b}$ show the pattern of the EVI over the two terrains, and it is clear from the figures that the overall pattern of the visibility indices is similar to the Golden Case. In fact, as indicated by the correlation coefficients, there is 97 percent and 82 percent overall correlation between the AVI and EVI of the Cairngorm and Isle of Man DEMs, respectively (Figures 6a and 7 a). The ridges and peaks have high visibility indices

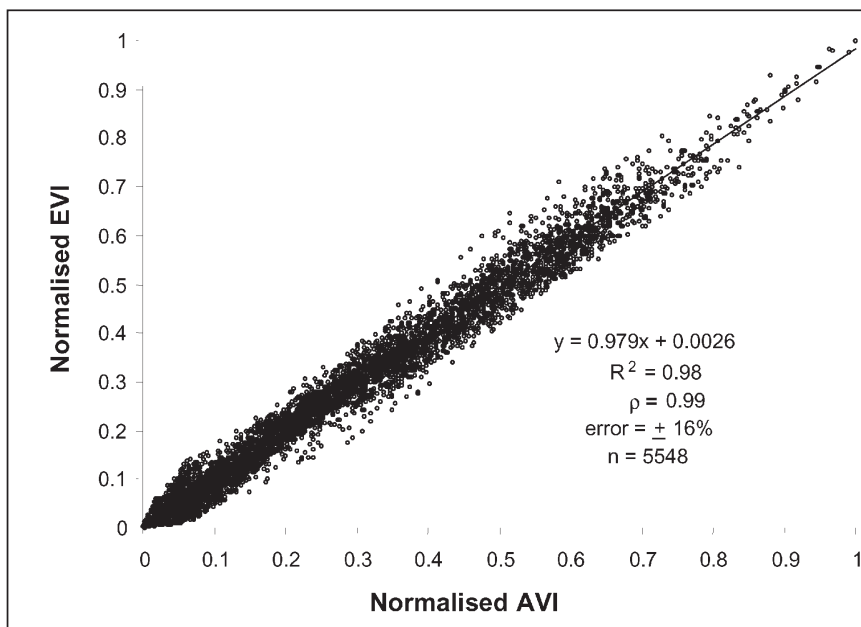

(a)

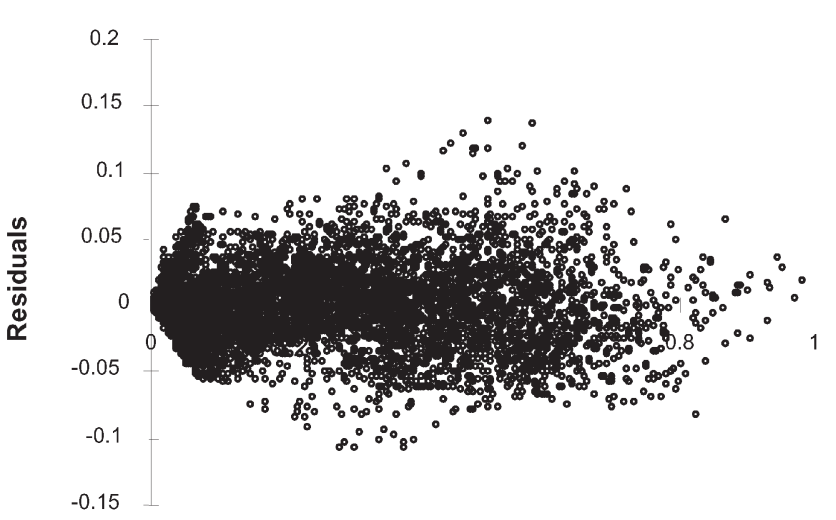

Predicted AVI

(b)

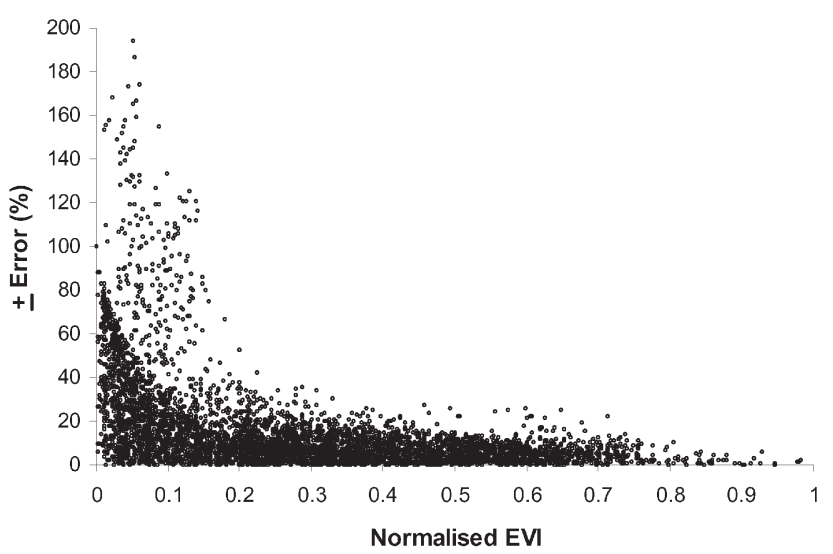

(c)

Figure 6. Uncertainty assessment in the EVI in the Cairngorm study area based on (a) AVI to EVI plot of all Cairngorm points, (b) residuals based on the linear regression between AVI and EVI, and (c) errors in the EVI. 


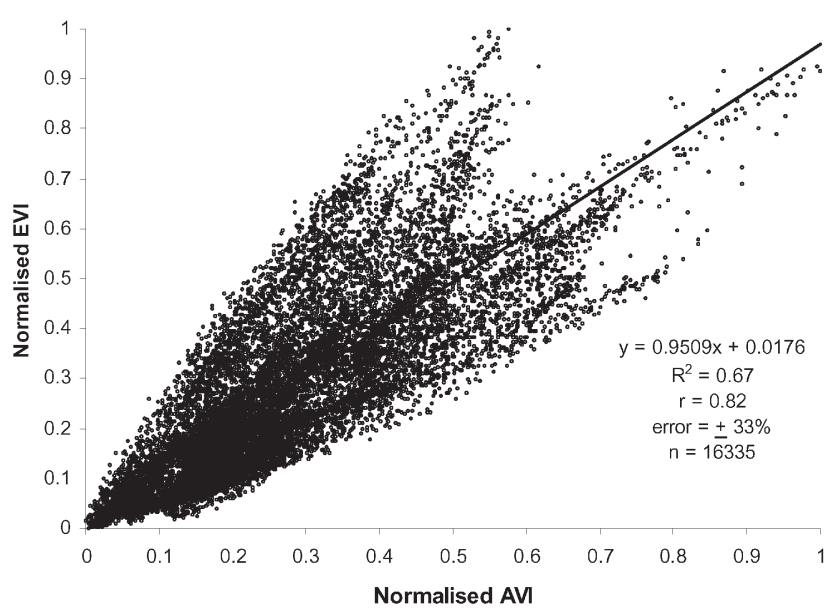

(a)

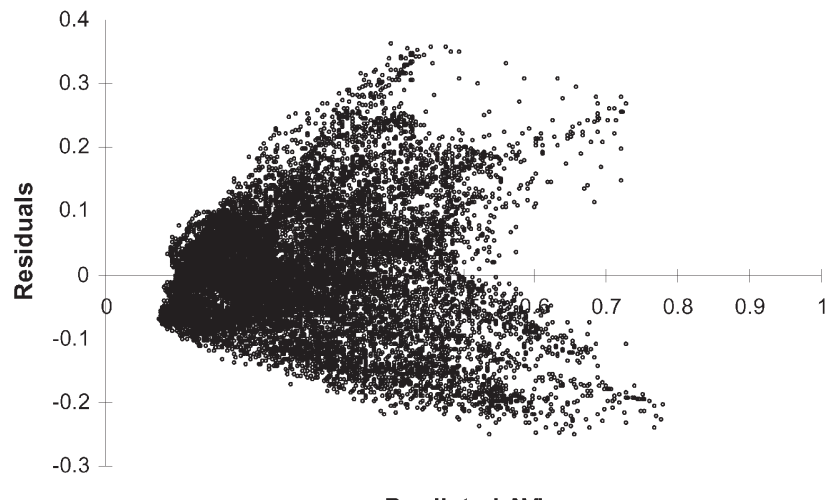

(b)

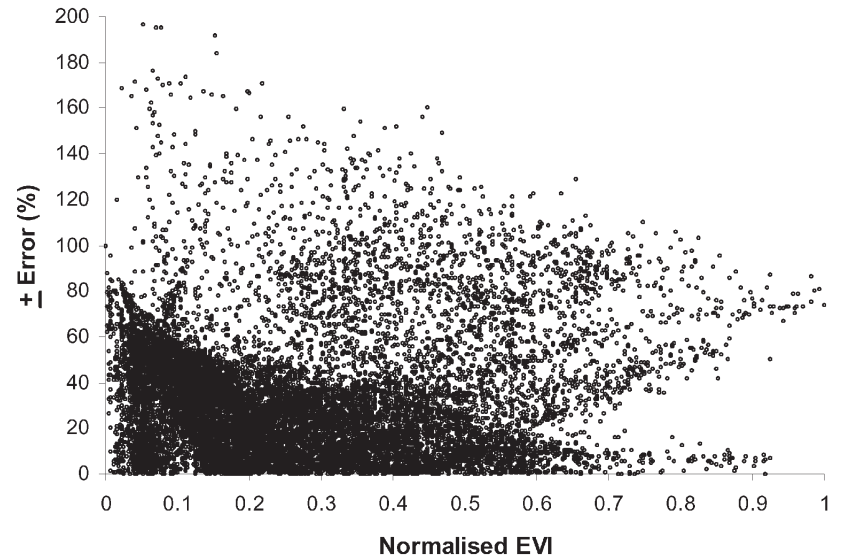

(c)

Figure 7. Uncertainty assessment in the EVI in the Isle of Man study area based on (a) AVI to EVI plot of all Isle of Man points, (b) residuals based on the linear regression between AVI and EVI, and (c) errors in the EVI. Note the distinct fork in both (a) and (b), indicating that the EVI has over estimated the visibility dominance of some points.

compared to the pits, passes, and channels. The plot of the AVI versus EVI for the Isle of Man DEM (Figure 7a) indicates that, in the case of some viewpoints, the optimization approach significantly overestimated their visibility dominance. A careful analysis of the spatial distribution of Figure 5b data revealed that these anomalous points are located around the main ridge structure in the central part of the DEM. The reason for this lies in the fact that the ridges make up a large proportion of the topographic features and therefore have a significantly better view of the topographic features (as also shown by Lee (1992)) compared to the other parts of the terrain. The plot of the residuals based on a linear regression supports this observation by the distinct fork-shape distribution of the residuals (Figure 7b). At the same time, it is evident from the range of the EVI that our optimized approach has significantly underestimated the visibility indices. The average error in the Cairngorm and Isle of Man DEMs are \pm 16 percent and \pm 33 percent, respectively. Further, Figures 6c and 7c show that the error varies according to the visibility dominance of the observer space, with the less dominant points having the bigger errors. The residuals versus the predicted AVI plots (Figures $6 \mathrm{~b}$ and 7b) reveal an interesting dichotomy. In the case of the Cairngorm study area, the residuals are uniform but, in the case of Isle of Man study area, the residuals are strongly related to the visibility index magnitude. An implication of this observation is that the regression between AVI and EVI should only be used as a basis for testing similarity (e.g., using the correlation coefficient) but not for modeling visibility magnitudes.

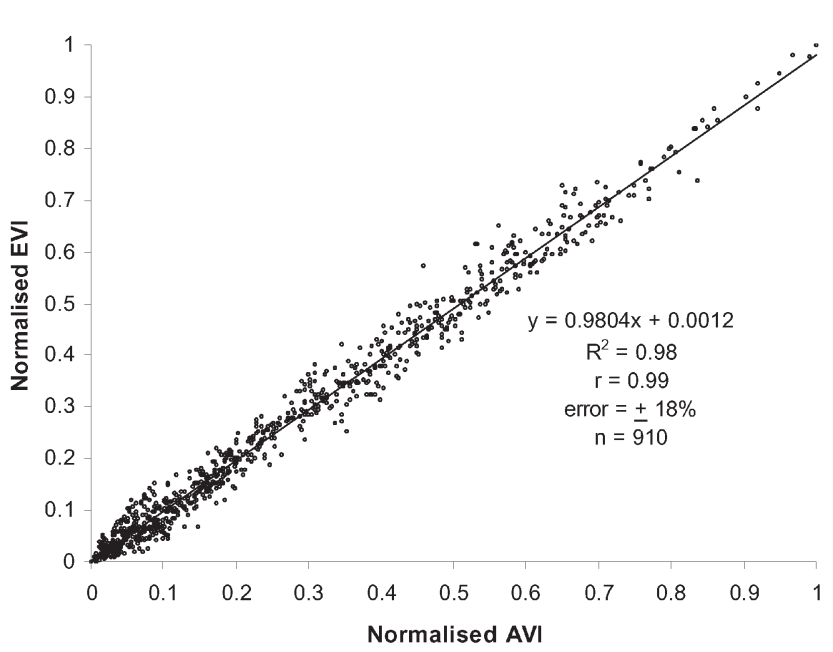

(a)

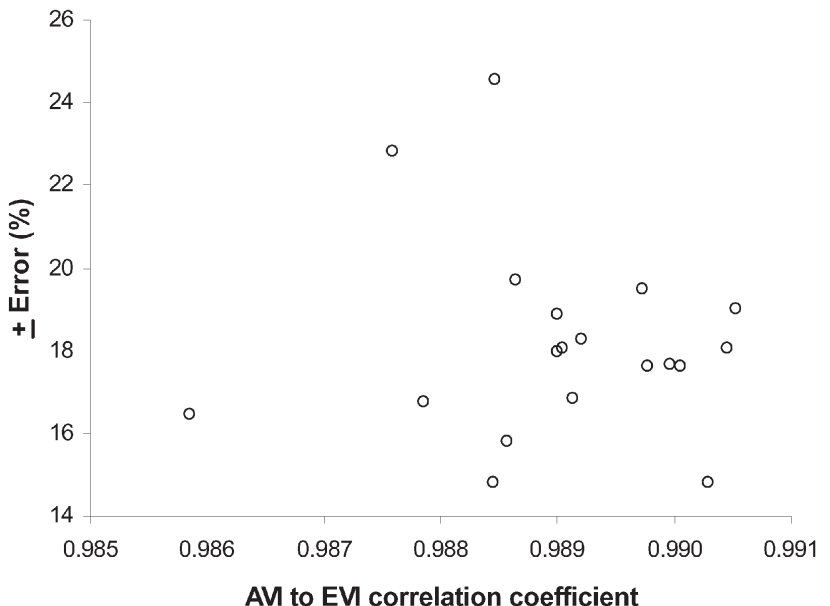

(b)

Figure 8. Uncertainty assessment in the EVI in the Cairngorm study area based on (a) AVI to EVI plot of the topographic features, and (b) AVI to EVI correlation coefficient versus errors at a set of random locations. 


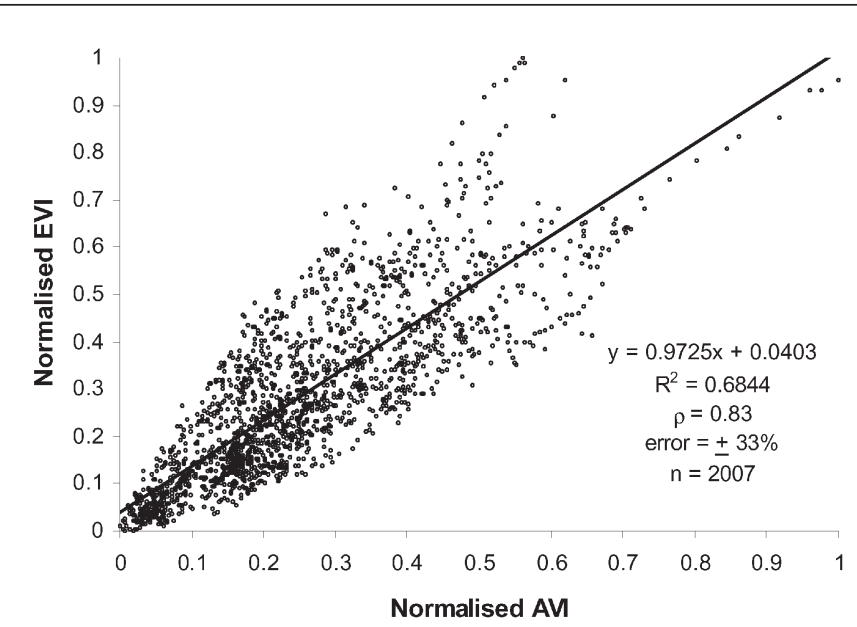

(a)

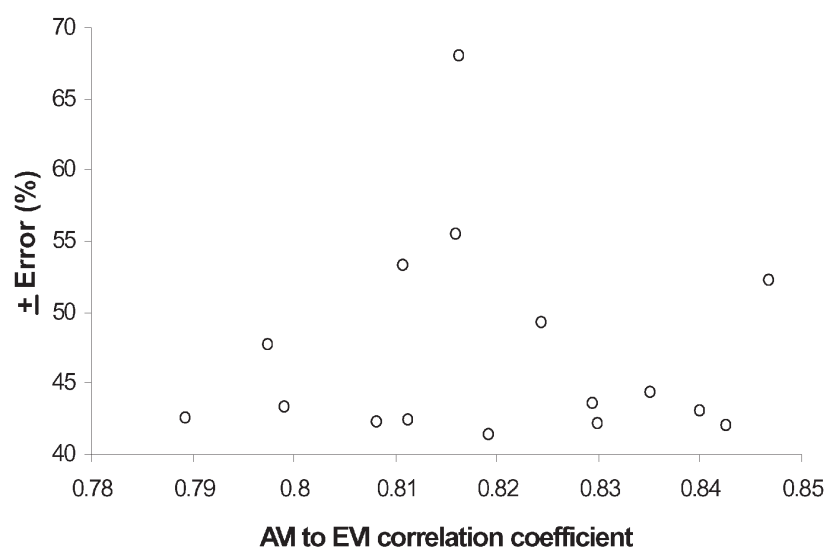

(b)

Figure 9. Uncertainty assessment in the EVI in the Isle of Man study area based on (a) AVI to EVI plot of the topographic features, and (b) AVI to EVI correlation coefficient versus errors at a set of random locations.

Based on Method 1 for uncertainty estimation, Figures 8a and 9a show the relation between the AVI and EVI of the topographic features in the Cairngorm and Isle of Man DEMs, respectively. The strong correlation coefficients of 0.98 (Cairngorm) and 0.83 (Isle of Man) suggest that the optimization has successfully achieved representing the overall visibility pattern. To perform a more exhaustive assessment, we generated 16 sets of 414 (unique number of EVI in the Cairngorm study area) and 19 sets of 479 (unique number of EVI in the Isle of Man study area) spatially distributed random points in the Cairngorm and Isle of Man study areas, respectively. We then calculated the correlation coefficient between the AVI and EVI for each of these sets of random points. Figures $8 b$ and $9 b$ show the wide variation in the quality of the estimated visibility pattern at various points on the terrain, thus supporting the exercise to validate the quality of the estimated visibility iteratively.

\section{Significance of the Fundamental Topographic Features}

Figures 10a and 11a show the comparison between the correlation coefficients and average approximation of the AVI and EVI calculated with the topographic feature targets and the random targets, at various target numbers. To begin with, note the considerably small number of targets compared to the total number of terrain points and yet the high correlation between the AVI and EVI of the observers. The figures show that

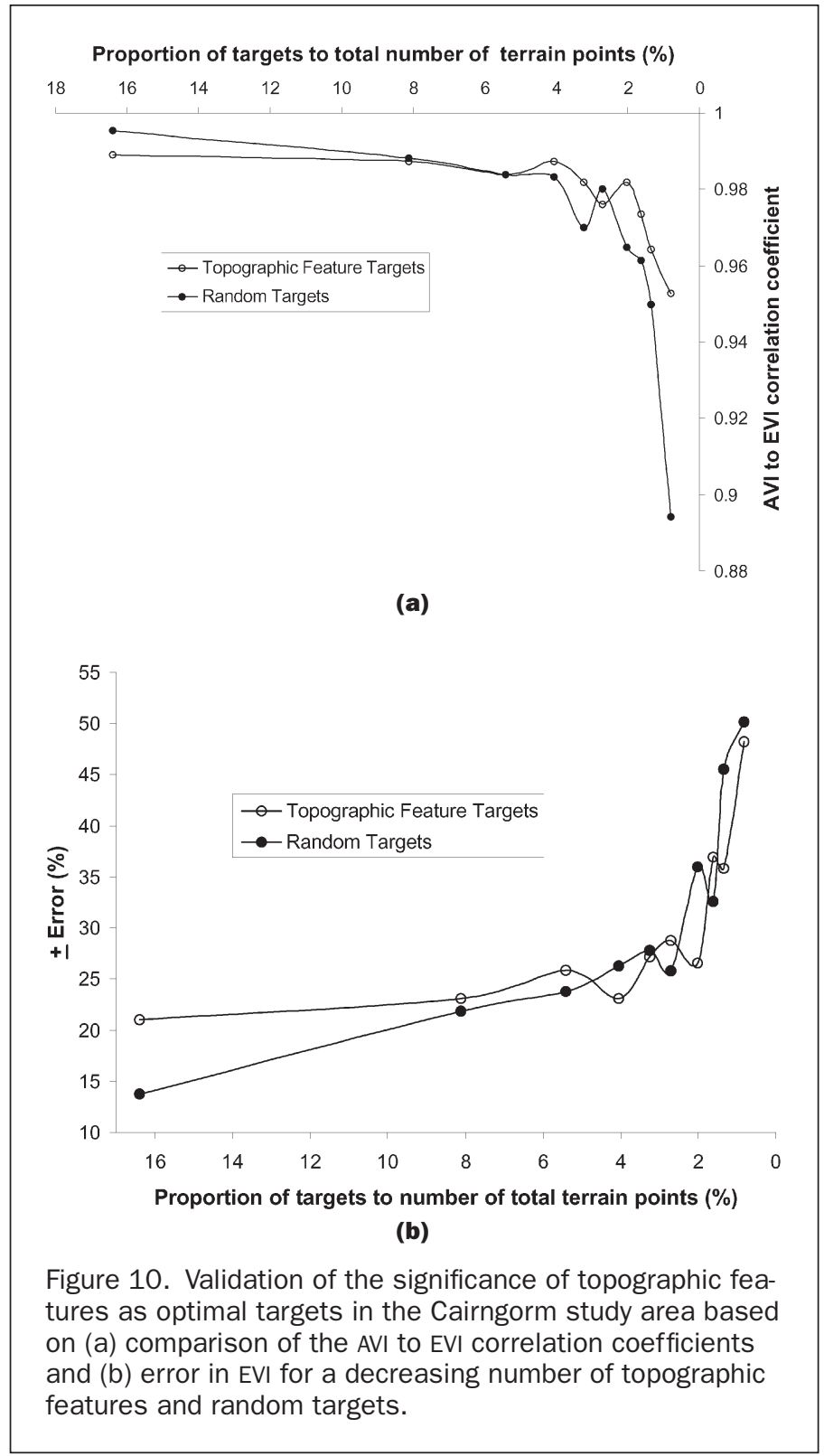

at high target numbers, by virtue of their wider spatial distribution, random targets could provide a better approximation of the visibility pattern than could the topographic features. However, as the number of targets is decreased, the quality of the approximation degrades rapidly in the case of random targets but, on the other hand, topographic features provide a more consistent and better approximation. This suggests that, at high target numbers, the better correlation between the AVI and EVI is a result of both the spatial distribution and the topographical significance of the reduced number of targets. However, at low numbers, the topographical significance will be a more useful basis for placing targets across the terrain. Therefore, it can be stated that one could reduce the number of the topographic features for the visibility computation for large terrains with a large number of topographic features, without the fear of losing any significant visibility pattern information. The plot of the errors in each case (Figures $10 \mathrm{~b}$ and $11 \mathrm{~b}$ ) essentially support the results based on correlation coefficients.

An interesting aspect of Figures 10a and 11a is the intersection of the topographic feature target and random target 

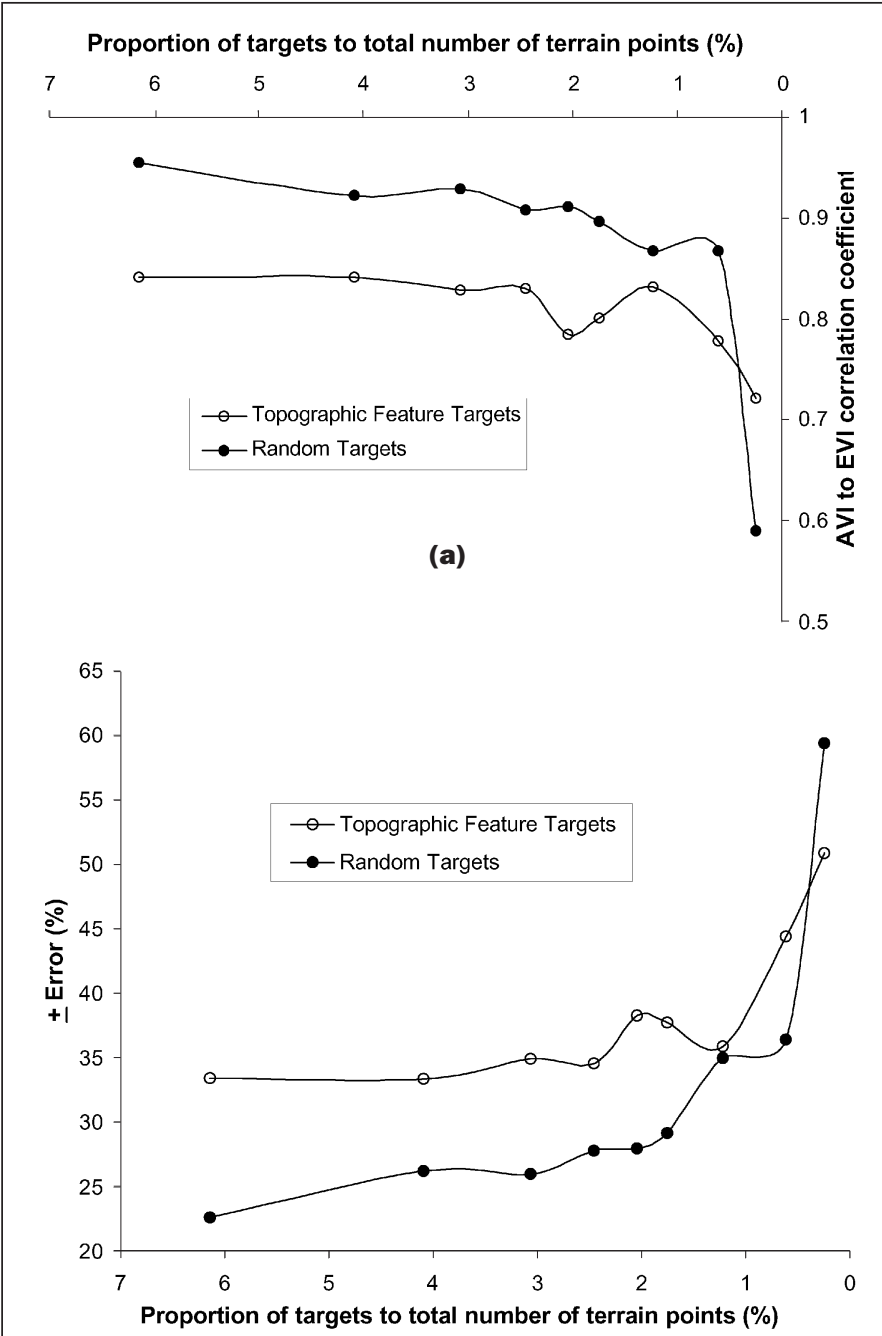

(b)

Figure 11. Validation of the significance of topographic features as optimal targets in the Isle of Man study area based on (a) comparison of the AVI to EVI correlation coefficients and (b) error in EVI for a decreasing number of topographic features and random targets.

correlation coefficient curves. It may indicate that in each terrain there are target numbers at which both the topographic feature targets and random targets could provide an equal level of spatial optimization. While there are situations in which this insight could be useful, for example, in deciding the optimal number of viewpoints for solving the time consuming minimum number of watchtowers problem (Lee, 1991), ironically it would not be possible to use this information without having done this iterative process.

\section{Optimization of Computation Time}

Figure 12 shows the linear relation between the CPU time usages versus the various magnitudes of visibility computations performed in the work. Computations here represent the product of the number of observers and the number of targets. The computation times for extracting the topographic features from the Cairngorm and Isle of Man DEMs in LandSerf were less than one second. As can be seen clearly, the time saved is substantial. However, the CPU time usage could be further optimized by combining the current approach with a Reduced Targets Strategy such as horizon culling.

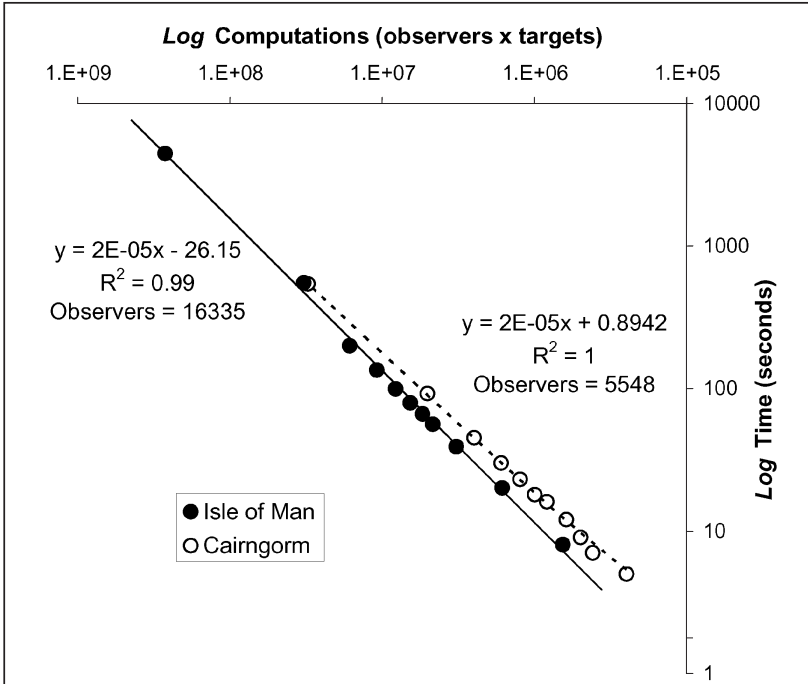

Figure 12. Linear relationship between the computation time and the computations.

\section{Conclusion and Future Work}

In this work, we have shown that the use of the fundamental topographic features as targets, as part of the Reduced Targets Strategy, can be used to decrease the visibility computation time substantially without any significant visibility information loss. This approach is especially useful for a fast approximation of visibility dominance in mountainous terrain. The reduced sampling of the targets on the terrain, however, introduces an uncertainty in the visibility indices of the observers on the terrain.

In the current work, the use of the correlation coefficient and the simple EVI to AVI ratio as measures of a visibility pattern quality and uncertainty provides only a global pattern matching, but visibility is a directional property. We anticipate developing ways in which we could estimate the visual integrity in our optimized approach. Although our observation that, at certain numbers, both topographic features targets and random targets would produce a similar quality of visibility estimation is based on thorough experimentation of the current study areas, experiments with other DEMs will be useful for fully validating this empirical observation.

The current work has also brought up a number of interesting questions, which could be investigated in future work. While the residuals between AVI and EVI in the case of the Cairngorm study area are uniform, in the case of Isle of Man study area, residuals appear to be dependent upon the visibility index magnitude. There can be many reasons for this anomaly, and one may even be able to model the non-uniform residuals in individual cases using a non-linear regression model. However, we believe it is more important to realize that visibility, as a property of terrain location, could not be modeled because it is derived only after an LOS test with other locations. Therefore, it is invariant of the local properties (e.g., elevation, slope, aspect) and global properties (e.g., geographic setting, i.e., faults, etc.) of a location. Thus, based on the current study, we believe that the regression between AVI and EVI only provides the information about the similarity or the amount of approximation.

Finally, two relatively straightforward extensions of the current work include (1) the combination of the Reduced $\mathrm{Ob}$ servers Strategy (e.g., horizon culling) and the proposed Reduced Targets Strategy for visibility index computation on very DEM and (2) the understanding of the effect of the topographic feature extraction scale on the computed visibility pattern. 


\section{Acknowledgments}

The author wishes to thank the two anonymous referees, Toshihiro Osaragi (Tokyo Institute of Technology), Mike Batty; Jeremy Morley; Daryl Lloyd (University College London), and Young-Hoon Kim (University of Sheffield) for providing critical feedback and materials support, which substantially improved the original manuscript. The DEM data are Crown Copyright and were provided by the Ordnance Survey (UK).

\section{References}

De Floriani, L., and P. Magillo, 1994. Visibility algorithms on triangulated terrain models, International Journal of Geographical Information Systems, 8(1):13-41.

De Floriani, L., P.K. Marzano, and E. Puppo, 1994. Line-of-sight communication on terrain models, International Journal of Geograph ical Information Systems, 8(4):329-342.

Evans, I.S., 1979. An Integrated System of Terrain Analysis and Slope Mapping, Final Report on Grant DA-ERO-591-73-G0040, University of Durham, Durham, United Kingdom, 192 p.

Fisher, P.F., 1991. First experiments in viewshed uncertainty-The accuracy of the viewshed area, Photogrammetric Engineering \& Remote Sensing, 57(10):1321-1327.

, 1992. First experiments in viewshed uncertainty: simulating the fuzzy viewshed, Photogrammetric Engineering \& Remote Sensing, 58(3):345-352.

, 1993. Algorithm and implementation uncertainty in viewshed analysis, International Journal of Geographical Information Systems, 7(4):331-347.

Franklin, W.M., C.K. Ray, and S. Mehta, 1994. Geometric Algorithms for Siting of Air Defense Missile Batteries, Technical Report on Contract No. DAAL03-86-D-0001, Battelle, Columbus Division, Columbus, Ohio, 116 p.

Franklin, W.M., 2000. Approximating visibility, Proceedings $1^{\text {st }}$ International Conference on Geographic Information Science, 28-31 October, Savannah, Georgia (National Center for Geographic Information and Analysis, University of California, Santa Barbara, California), pp. 126-138.

Greysukh, V.L., 1967. The possibility of studying landforms by means of digital computers, Soviet Geographer, 137-149.

Jenson, S.K., and J.O. Domingue, 1988. Extracting topographic structure from digital elevation data for geographic information systems analysis, Photogrammetric Engineering \& Remote Sensing, 54(11):1593-1600

Jennes, J., 2001. Random Point Generator Extension v.1.1 for ArcView http://jennessent.com/arcview/random_points.htm (last accessed 16 November 2001).
Kim, Y-H., S.S. Rana, and S. Wise, 2002. Exploring multiple viewshed analysis using terrain features and optimisation techniques, Proceedings of the GISRUK 2002, 03-05 April, University of Sheffield, United Kingdom, pp. 16-21.

Lee, J., 1991. Analyses of visibility sites on topographic surfaces, International Journal of Geographical Information Systems, 5(3):413-429.

1992. Visibility dominance and topographical features on digital elevation models, Proceedings of the Fifth International Symposium on Spatial Data Handling, August, Charleston, South Carolina (Humanities and Social Sciences Computing Lab, University of South Carolina, Columbia, South Carolina), 2:622-631.

O’Sullivan, D., and A. Turner, 2001. Visibility graphs and landscape visibility analysis, International Journal of Geographical Information Systems, 15(3):221-237.

Peucker, T.K., and D.H. Douglas, 1975. Detection of surface-specific points by local parallel processing of discrete terrain elevation data, Computer Graphics Image Processing, 4:375-387.

Takahashi, S., T. Ikeda, Y. Shinagawa, T.L. Kunii, and M. Ueda, 1995. Algorithms for extracting correct critical points and constructing topological graphs from discrete geographical elevation data, The International Journal of the Eurographics Association, 14(3): C181-C192.

Wang, J., K. White, and G.J. Robinson, 2000a. Estimating surface net solar radiation by use of Landsat-5 TM and digital elevation models, International Journal of Remote Sensing, 21(1):31-43.

Wang, J., G.J. Robinson, and K. White, 2000b. Generating viewsheds without using sightlines, Photogrammetric Engineering \& Remote Sensing, 66(1):87-90.

Weibel, R., and G. Dutton, 1999. Generalising spatial data and dealing with multiple representations, Geographical Information Systems: Volume 1-Principles and Technical Issues (P.A. Longley, M.F. Goodchild, D.J. Maguire, and D.W. Rhind, editors), John Wiley and Sons Inc., New York, N.Y., pp. 125-155.

Wood, J., 1998. Modelling the continuity of surface form using digital elevation models, Proceedings of the Eighth International Symposium on Spatial Data Handling, 11-15 July, Vancouver, British Columbia, Canada (Department of Geography, Simon Fraser University, Vancouver, British Columbia, Canada), pp. 725-736. 1999. Visualisation of scale dependencies in surface models, URL: http://www.soi.city.ac.uk/ jwo/ica99 (last accessed 16 November 2001).

(Received 05 December 2001; accepted 29 October 2002; revised 19 November 2002) 